\title{
Neural correlates of language aptitude during a lexical semantic categorization task
}

Olga Kepinska ${ }^{1,2}$, Esli Struys ${ }^{2}$

${ }^{1}$ Vrije Universiteit Brussel, TALK, Belgium

${ }^{2}$ Leiden University Centre for Linguistics, the Netherlands

https://doi.org/10.36505/ExLing-2012/05/0019/000225

\begin{abstract}
In an fMRI experiment language aptitude, a specific talent for learning languages measurable by tests, has been used as an independent variable and brain activity elicited by a semantic categorization task has been measured. Significant differences in brain functionality have been observed between groups in two comparisons.
\end{abstract}

Key words: neurolinguistics, language aptitude, semantic categorization

\section{Introduction}

Language aptitude, a specific talent for learning languages, is considered to be one of the most robust predictors of language learning success (Dörnyei \& Skehan, 2003; Ellis, 2008; Sawyer \& Ranta, 2001). Four subcomponents of language aptitude are traditionally distinguished: rote learning memory, phonemic coding ability, inductive language learning ability and grammatical sensitivity (cf. Carroll, 1981; Dörnyei \& Skehan, 2003; Ellis, 2008). Most aptitude tests underscore the multicomponential nature of language aptitude, e.g. the Llama Language Aptitude Test (LLAMA) (Meara, 2005) consists of four sub-tests: (1) a vocabulary learning task, (2) a test of phonetic memory, (3) a test of soundsymbol correspondence and (4) a test of grammatical inferencing. Despite the recent technological advances in the domain of neurolinguistics, it is still largely unknown how success in foreign language learning, as measured by such tests, can be accounted for in terms of its neural correlates and how the fact that some people are faster and better language learners than others can be associated with the brain functionality on language tasks. The aim of this research was thus to establish the neural correlates of language aptitude during a lexical semantic categorization task (SCT).

\section{Experimental procedures}

\section{Participants}

24 right-handed healthy Dutch-French bilinguals (11 female, mean age 20.54; SD 1.96) took part in the study. The research protocol was approved by the ethical commission of the University Hospital of Jette.

ExLing 2012: Proceedings of 5th Tutorial and Research Workshop on Experimental Linguistics, 27-29 August 2012, Athens, Greece 


\section{Design}

Language aptitude test and semantic categorization task

The LLAMA test (Meara, 2005) was administered on a Personal Computer to each participant individually and they received written instructions in Dutch prior to each part. Target items in the SCT consisted of 156 stimuli: 39 Dutch and 39 French animals, and 39 Dutch and 39 French non-animals. The items were matched for frequency, number of syllables, length, lexical neighbours and interlingual homographs and homophones. The stimulus duration of $2200 \mathrm{~ms}$. was fixed for all participants. The task's trials were synchronized with the scanning trials and participants' reaction times and accuracy levels were measured.

\section{Data analysis and image acquisition}

Language aptitude test

Scores for each part of the LLAMA test range between 0 and 100. A Principal Components Analysis with VARIMAX rotation of these scores was conducted in order to reveal the factors underlying the larger construct of aptitude. Three tests (vocabulary learning, sound recognition and sound symbol correspondence) loaded highest on Component $1(\lambda=.852, \lambda=.656$, and $\lambda=.712$ ). Grammatical inferencing loaded on a separate component. Due to the nature of the tasks, the two factors were interpreted as 'language aptitude on word level' (Component 1) and 'language aptitude on sentence level' (grammatical inferencing). Subsequently, based on their scores a distinction was made between above and below average performers on each factor.

Image acquisition and processing

The images were acquired with a 3 Tesla Philips Achiva with an 8 channel head SENSE coil. Activations were detected by means of BOLD sensitive T2* weighted fMRI and a T1 weighted anatomical scan was used to project the fMRI images. Repetition time was $3000 \mathrm{~ms}$ and the echo-time was 35 ms. 22 slices with a thickness of $4 \mathrm{~mm}$ were obtained over 130 dynamic scans. The anatomical scan consisted of 100 slices with a thickness of $2 \mathrm{~mm}$ and was used to co-register the functional scans. The acquired data were subsequently analyzed with SPM5 software (The Wellcome Institute of Neurology, London, UK). Functional images were spatially realigned, coregistered with the anatomical data, spatially normalized to standard stereotactic MNI space, and smoothed with an isotropic 8-mm full-width at half-maximum Gaussian kernel. 
Neural correlates of language aptitude during acategorization task

\section{Results}

\section{Imaging Data}

Although no significant differences in either of the performed group statistics were found for the behavioural data, significant differences between the groups in brain activations during the task were found. Whereas the group with below average language aptitude on word level displayed more brain activity during the task, the reverse could be observed for the effect of language aptitude on sentence level.

The difference in brain activations between the groups were found in a two sample analysis. The participants with below average language aptitude on word level displayed more brain activity during the task. The activated regions included the left Thalamus $(\mathrm{p}=0.003$, uncorrected $)$ and right Insula (BA 13) $(\mathrm{p}=0.015$, uncorrected).

A difference in activations was found only when the activations of the below average 'sentence level' language aptitude performers were subtracted from the above average 'sentence level' language aptitude performers. The analysis reported increased activations in the right Middle Temporal Gyrus (BA 39) $(\mathrm{p}=0.005$, uncorrected), right Posterior Cingulate (BA 31) $(\mathrm{p}=$ 0.000 , uncorrected) and left Superior Frontal Gyrus (BA 9) $(p=0.041$, uncorrected).

\section{Discussion}

Using an SCT and an event-related fMRI, effort was made to unveil functional brain differences typical for high and average performers on two factors of a language aptitude test involved in language switching. There were significant differences in brain functionality when two independent comparisons of the acquired data were conducted. The left thalamus activated in the case of the below average performers on the 'word level' language aptitude component has been previously tied to grammatical (Wartenburger et al., 2003) and morphosyntactic (Fabbro et al., 1997) processing of the second and less-exposed language; the insular cortex has been established to support lexical-semantic processes (Friederici et al., 2003). In the current study these regions were related to language switching by bilinguals with less skill on the lexical component of language aptitude, suggesting that these regions can be tied to language aptitude related neuroanatomical differences. The regions activated in the case of the above average 'sentence level' language aptitude group were previously identified to be involved in semantic memory processing (middle temporal gyrus) (Onitsuka et al., 2004), recall of previously memorized words from temporal cues (posterior cingulate) (Fujii et al., 2002) and to working memory (Du Boisgueheneuc et al., 2006). The exact relationship between these regions 
and high level of analytical skills remains a question to be answered in future research specifically investigating syntactic processing.

\section{Acknowledgements}

Thanks to our supervisor Piet Van de Craen and to Ghazal Mohades, Jessica Schillaci, Lilith Van Biesen and Thomas Somers.

\section{References}

Carroll, J.B. 1981. Twenty-five years of research in foreign language aptitude. In Diller K. (Ed.), Individual Differences and Universals in Language Learning Aptitude. Newbury House, pp. 83-118.

Dörnyei, Z., Skehan, P. 2003. Individual differences in second language learning. In Doughty, C.J. Long, M.H. (Eds.), Handbook of second language acquisition (pp. 612-630). Oxford: Blackwell.

$\mathrm{Du}$ Boisgueheneuc et al. 2006. Functions of the left superior frontal gyrus in humans: a lesion study. Brain, 129, 3315-3328.

Ellis, R., 2008. The Study of Second Language Acquisition (2nd edition), Oxford: Oxford University Press.

Fabbro F, Peru A, Skrap 1997. Language disorder in bilingual patients after thalamic lesions. Journal of Neurolinguistics, 10(4): 347-367.

Friederici, A., Rüschemeyer, S., Hahne, A., Fiebach, C. 2003. The Role of Left Inferior Frontal and Superior Temporal Cortex in Sentence Comprehension: Localizing Syntactic and Semantic Processes. Cerebral Cortex 13:170-177.

Onitsuka et al. 2004. Middle and Inferior Temporal Gyrus Gray Matter Volume Abnormalities in Chronic Schizophrenia: An MRI Study. Am J Psychiatry 161(9): 1603-1611

Fujii et al. 2002. The role of the basal forebrain in episodic memory retrieval: a positron emission tomography study. NeuroImage 15(3):501-8.

Meara, P.M. 2005. Llama Language Aptitude Tests. Swansea: Lognostics.

Wartenburger I, Heekeren HR, Abutalebi J, Cappa SF, Villringer A, Perani D. 2003. Early setting of grammatical processing in the bilingual brain. I, 37(1): 159-170. 